\title{
Quantifying the Relative Difficulty of Design Engineering Term Projects
}

\author{
George Platanitis and Remon Pop-Iliev \\ University of Ontario Institute of Technology \\ george.platanitis@uoit.ca \\ remon.pop-iliev@uoit.ca
}

\begin{abstract}
Are we aware of how challenging the design engineering projects are that we assign to engineering students? This paper emphasizes the pedagogical importance of evaluating the dynamics of the iterative tasks pertaining to assigned design engineering term projects in academia for reasons of quantifying, balancing and adjusting their relative level of difficulty. Methodologies exist in industry for large projects, such as for example the design of the various systems of automobiles or aircraft, to assist engineers and project managers with the efficient planning of such tasks, possibly eliminating some of the iterations altogether, as well as ordering tasks (where eliminating iteration is impossible) to minimize duration of coupled tasks. The Design Structure Matrix (DSM), which along with its extension - the Work Transformation Matrix (WTM), have proven to be useful methodologies for predicting convergence of iteration, as well as in figuring out which coupled tasks will require several iterations to reach a technical solution. In this context, a DSM/WTM-based method is proposed that educators within an engineering curriculum could apply to pre-determine the difficulty of the design projects they assign and could adjust them, if necessary, so to be commensurate to the current academic level of the respective learners.
\end{abstract}

\section{Introduction}

Often design engineering educators create their own term design project problems/topics to be assigned to students during a particular course offering within the undergraduate engineering curriculum. Thus, it should be relatively easy to plan the tasks and their order for the design process of a $1^{\text {st }}$-year design project which requires few steps, with very few tasks being coupled. Even then, with perhaps only a small number of tasks requiring iteration, these tasks can be completed fairly quickly. However, this is normally not the case with many $2^{\text {nd }}-, 3^{\text {rd }}$ - and $4^{\text {th }}$-year level design projects, which are more complex, are often of multidisciplinary nature, and require quite a few tasks to be completed. Very often such tasks cannot be sequenced in an order so that one is completed after another, but rather, they have to be performed concurrently. Relationships between such tasks can be quite complex, with each coupled task relying on information from each other. This directly affects the increase of the relative difficulty of the design engineering challenge being assigned to students. The question becomes: could we quantify this level of difficulty in a meaningful way ahead of time so that we can compare and rank different projects in terms of such difficulty. In this paper, the use of DSM methodologies in this context is examined for a $3^{\text {rd }}$ year engineering design project as an example assigned at UOIT. Using an eigenvalue and eigenvector analysis on the WTM, the modes of the design iteration can be identified and the tasks that contribute the greatest amount of work to each mode before the design process converges to an acceptable design. Using such information, educators can quantify with certainty the relative level of difficulty of a design project they are about to assign, while students can systematically determine tasks where either iteration speed can be increased or they determine ways to reduce the number of iterations.

\section{Background}

Research in the last several years has focused on methods that provide for better organization of design tasks. Here, matrix representation (i.e., Design Structure Matrix) is used to define the sequence of and the technical relationships between design tasks, and then finding alternative sequences or task definitions to improve the design process [1]. The Design Structure Matrix (DSM) has been further extended to make use of the fully coupled portion, which is called the Work Transformation Matrix (WTM) [2]. Here, the WTM not only relates dependencies between tasks, but also includes the strength of this dependence, in terms of the probability of rework of a task. Rework is the required repetition of a task when 
it has originally been attempted with imperfect information, and needs to be "reworked" when new or modified information becomes available for that task. Further, using an eigenstructure analysis, design modes were identified for a camera design and a brake system design. The design modes determine the controlling features of the design iteration.

Smith and Eppinger [3] further use the design structure matrix in order to predict the expected duration of the iterative solution process, and use optimizing algorithms and the reward Markov chain method to find the best order for coupled design tasks.

In Cronemyr, et al [4], the authors use DSM and WTM methods to predict the effect of improvements to certain aspects of the development process. They introduce the concept of total process time and simulated to-be/as-is ratio, where the latter is a sensitivity measurement of the total process time with suggested improvement versus the original process time. Suggested improvements include methods for speeding up iterations or avoiding some iterations altogether. Using eigenvalue analysis, the slowest converging mode of the iteration is identified, and from the corresponding eigenvector, which task has the largest contribution. Improvements can then be suggested to speed up the task, should it be among the tasks with the longest completion times.

In another application of DSM and WTM methods, the design process is represented as a discrete statespace system, and using well established methods of dynamic analysis techniques to investigate and predict the dynamics of the concurrent design process [5]. Using Smith and Eppinger's camera design example [2], the author is able to predict the required iterations for each of the design tasks, proving that for a given task dependency, the required iteration is dependent

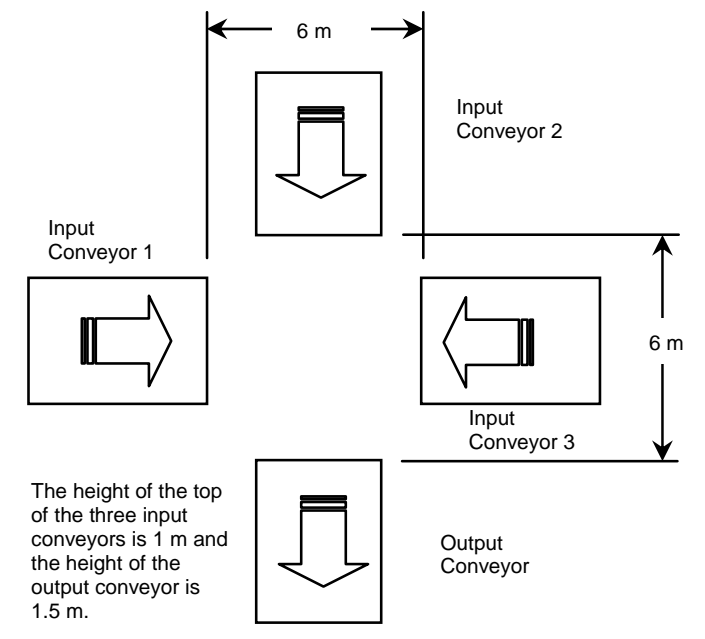

Figure 1. Conveyor belt layout and scaling pertaining to tire manipulator design project. on the eigenvalues of the WTM. The methodology utilized took advantage of modal analysis to determine the stability of each of the design iteration modes. The methodology of Ref. 5 also overcame deficiencies of previous methods performing stability analysis which used continuous state-space models [6].

\section{Example: A Third-Year Design Project}

Engineering students enrolled in mechanical-based programs at UOIT in the $3^{\text {rd }}$ year undertake an integrated design project between two courses which combines concepts of $\mathrm{CAD} / \mathrm{CAM} / \mathrm{CAE}$ design principles (3D Scanning, Solid Modelling, Rapid Prototyping, Finite Element Methods, etc.) with kinematic and dynamic analysis of machines.

Working in groups of about 4-5, students were charged with the task of designing a mechanism for handling and manipulating three different tire sizes, to be picked up from three input conveyor belts oriented at 90 degrees to each other, and placed on an output conveyor (see Fig. 1). The mechanism must be designed with a factor of safety between 2.5 and 5 (anything greater is considered over-engineered), and must be able to handle tires weighing no more than 50 $\mathrm{kg}$ each. In addition to the CAD design, students must build and demonstrate a prototype of their design using Lego Mindstorms ${ }^{\circledR}$. Students were required to design the actual manipulator in CAD using real parts and to proper size (not to simply enlarge the Lego pieces).

The entire design process for the tire manipulator may be broken down into several tasks. They are listed below in a suggested order, though many of the tasks will be coupled to some degree:
A. Determine specifications
B. Design concept
C. Design arm
D. Design arm joints
E. Design grip mechanism
F. Design rotating base
G. Finite Element Analysis
H. Dynamic simulation
I. Factor of safety
J. Failure mode analysis
K. Build prototype
L. Evaluate prototype

Regardless of the complexity of a design project, students often carry out the design effort in a somewhat disjointed order, while focusing on tasks at the wrong time. In this example, students have been found to become fixated with building a working 
prototype first, and waiting until the end to develop the CAD model and the associated analyses. Also, although students make use of various organizational tools, such as logbooks and Gantt charts, etc. [7], they do not always comprehend just how many subtasks are involved with a relatively complex design, or to what degree task coupling occurs. Also, such flow charts do not model task iterations. Introducing DSM methods may serve well the instructors in terms of evaluating and quantifying the difficulty of the design challenge at hand as well as may help students further with task organization.

\section{Design Structure Matrix (DSM)}

The tasks for the tire manipulator design process are then organized into a matrix structure. Developed originally by Steward [8], the matrix represents the relationships between all the tasks.

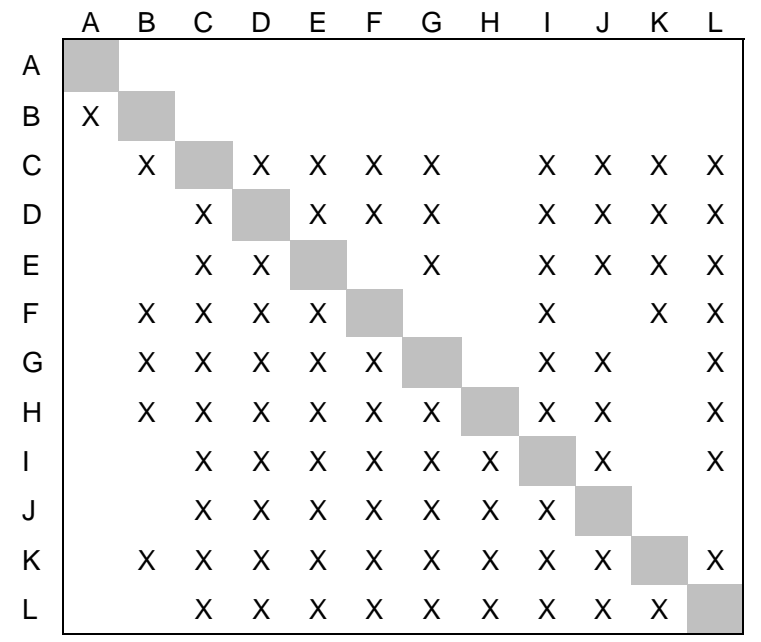

Figure 2. DSM matrix representation for tasks to be performed in tire manipulator design.

An example of the DSM for the tire manipulator design is shown in Fig. 2. The tasks are listed down the left side and along the top, in some logical 'order' that they are to be completed (if there was no coupling between tasks). In this representation, an ' $\mathrm{X}$ ' is placed in the off-diagonal boxes to show the dependency of a task with another. For example, an ' $\mathrm{X}$ ' in box I-F (row I, column F) means that Task I is dependent on information from the completion of Task F. Ideally, one would prefer to have ' $\mathrm{X}$ 's in only the lower triangle, indicating a feedforward direction of task dependencies (that is, an upstream task will not be repeated; once complete, the next downstream task that depends on it will start). Unfortunately, complex projects will have some amount of feedback involved between downstream and upstream tasks (' $\mathrm{X}$ 's in the upper triangle), meaning that upstream tasks will have some amount of rework necessary.

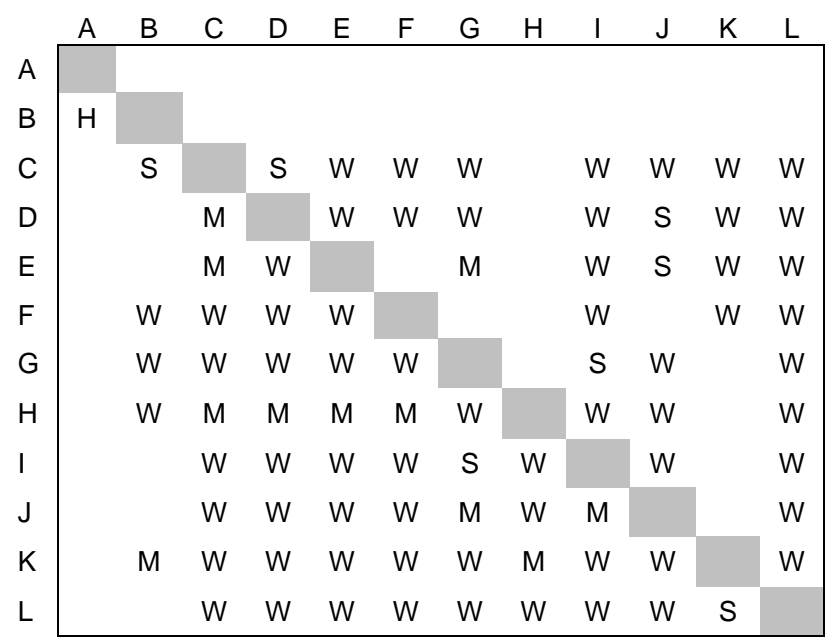

Figure 3. WTM matrix representation for tasks to be performed in tire manipulator design.

\subsection{Work Transformation Matrix (WTM)}

A further extension of the DSM is the WTM, which shows the strength of the dependencies between tasks [1-5]. Several assumptions need to be made to perform the analysis [2]:

- All tasks are completed at every stage (fully parallel iteration).

- Rework is a function of work done in the previous iteration stage.

- Work transformation parameters are time invariant.

For this study, the strength of the dependencies will be simple [2], and assigned the following numerical values: $0.5,0.25$, and 0.05 for strong (S), medium (M), and weak (W) dependencies respectively. Alternatively, dependence information can be defined as [1]:

- Strong - information required from previous task to begin task.

- Medium - information required to end task.

- Weak - information required to check result compatibility.

- Zero - no information required

More rigorous study of the tasks would be required to obtain precise values of their dependency strengths. This dependency strength can be looked at as the amount of work (in terms of a percentage of time to 
determine a parameter in the original iteration) that the upstream task creates for a downstream task that depends on the upstream task's information. For the tire manipulator, the WTM is that represented in Fig. 3. Using the numerical version of the coupled block of the WTM (tasks C to L), the eigenvalues and eigenvectors will be determined. From this information, will contribute the greatest amount of work will be determined.

\subsection{Mathematical Formulation}

At each iteration stage, an amount of work will have been completed for each task. The idea of the work vector is introduced here, and the elements of the work vector indicate how much work is left for each task to complete. Initially, the work vector is a column vector of ones, that is, all of the work remains for each task. As the design process progresses from iteration stage $t$, the work remaining at the next stage is given by $[2,5]$ :

$$
\mathbf{u}_{t+1}=\mathbf{A} \mathbf{u}_{t}
$$

where $\mathbf{u}_{t}$ is the work vector at stage $t$ and $\mathbf{A}$ contains the strength dependencies of the WTM, and each $a_{i j}$ element $(i \neq j)$ implies that doing a unit of work on task $j$ creates $a_{i j}$ units of work on task $i$. Each element in the work vector represents the work remaining for the task it is associated with at iteration stage $t$. From the initial work vector $\mathbf{u}_{0}$, the remaining work at stage $t$ may alternatively be given by:

$$
\mathbf{u}_{t}=\mathbf{A}^{t} \mathbf{u}_{0}
$$

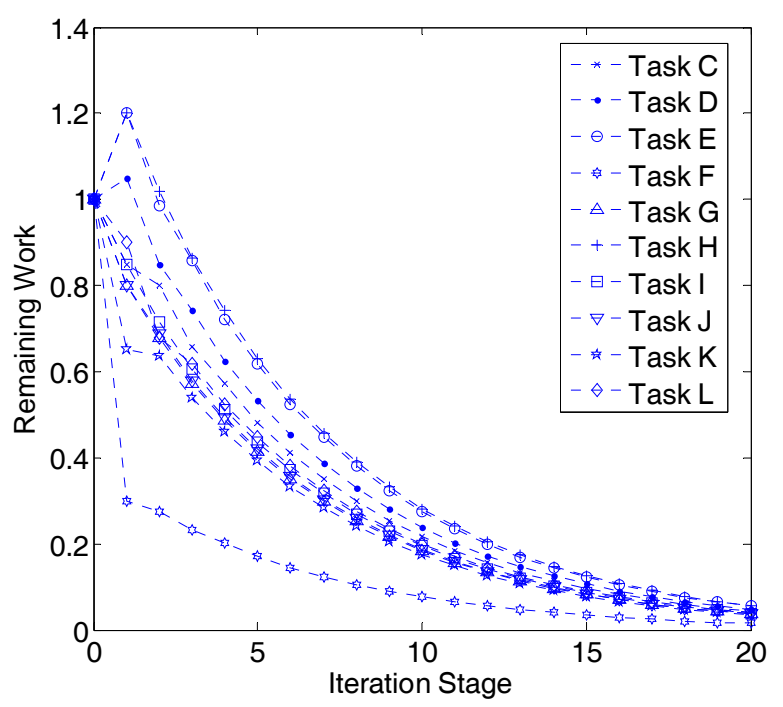

Figure 4. Remaining work for each task in tire manipulator design process.
Summing up all the work vectors after $M$ iterations gives the total work vector for the entire design process:

$$
\mathbf{u}_{\text {tot }}=\sum_{t=0}^{M} \mathbf{u}_{t}=\sum_{t=0}^{M} \mathbf{A}^{t} \mathbf{u}_{0}=\left(\sum_{t=0}^{M} \mathbf{A}^{t}\right) \mathbf{u}_{0}
$$

As presented in Ref. 5, treating the dynamics of the design process as a discrete-time system is logical. Just as the state variables of a discrete-time system are computed at discrete intervals, for the design process model, the work vector is computed at discrete intervals (the iterations themselves). Eq. 1 resembles a discrete system. Further, the dynamics of the discrete system represented by Eq. 1 can be analyzed as a continuous time system being sampled at intervals of 1 (i.e., iterations take place at step 1, 2, 3, etc.). Details for the stability of discrete systems can be found in many textbooks (eg., Ref. 9).

To determine the stability of the iterative design process, one can look at the eigenstructure of $\mathbf{A}$. If $\mathbf{A}$ has linearly independent eigenvectors, then $\mathbf{A}$ can be decomposed as:

$$
\mathbf{A}=S \Lambda S^{-1}
$$

where $\Lambda$ is a diagonal matrix containing the eigenvalues of $\mathbf{A}$ and $S$ is the eigenvector matrix. A discrete time system is stable if the magnitude of its eigenvalues lie within the unit circle on a complex plane [9].

It is important to note that if the design process (as represented by the WTM model) is stable, then it will converge to a technically feasible solution given the specifications of the product to be designed. A nonconverging process would mean that a feasible solution does not exist, or that the designers are unwilling to compromise in order to come up with a solution.

Table 1. Eigenvalues for WTM Model of Tire and Wheel Manipulator

\begin{tabular}{|c|c|c|}
\hline Mode & Eigenvalue & $\begin{array}{c}\text { Eigenvalue } \\
\text { Magnitude }\end{array}$ \\
\hline 1 & 0.8512 & 0.8512 \\
2 & -0.5029 & 0.5029 \\
3 & -0.3440 & 0.3440 \\
4 & 0.2571 & 0.2571 \\
5 & $-0.0626 \pm 0.1967 \mathrm{i}$ & 0.2064 \\
6 & -0.1568 & 0.1568 \\
7 & $0.0350 \pm 0.0304 \mathrm{i}$ & 0.0464 \\
8 & -0.0496 & 0.0496 \\
\hline
\end{tabular}




\section{Results}

For the WTM of Fig. 3, the eigenvalues and eigenvectors are computed. Also, the remaining work for each task at each iteration is computed and plotted in Fig. 4. As can be seen, the design process for the tire manipulator is stable, with a feasible design possible after approximately $15-20$ iterations. It is also significant to note that Tasks $\mathrm{D}, \mathrm{E}$, and $\mathrm{H}$ (design of the arm joints, grip, and dynamic simulation, respectively) show that after the first iteration, more work remains at this stage than when the design process originally started; however, the work remaining otherwise decreases (monotonically) with each subsequent iteration, a sufficient condition for stability of the design process [5].

Determining the eigenvalues of the WTM reveals that there are 8 design modes (see Table 1). Note that the magnitude of each eigenvalue is $<1$, meaning that each design mode is stable. The modes are ranked based on the magnitude of the eigenvalues. The first mode has the largest magnitude eigenvalue, and will have the slowest dynamics $[2,5]$. This mode will have an eigenvector that is strictly positive [2], and will be the most obvious to interpret.

According to the Perron-Frobenius Theorem, the largest magnitude eigenvalue of A (a non-negative matrix) will be strictly positive and will have a strictly positive eigenvector (the only positive eigenvector) associated with it. Table 2 shows the eigenvectors of the two modes with the largest eigenvalues as per Table 1.

For clarity: The Perron-Frobenius Theorem states that a matrix with nonnegative entries has at least one nonnegative eigenvalue of maximal absolute value and a corresponding eigenvector with nonnegative components. The importance of the Perron-Frobenius theorem stems from the fact that eigenvalue problems on these types of matrices frequently arise in many different fields of science and engineering such as steady state behaviour of Markov chains, power control in wireless networks, commodity pricing models in economics, population growth models, Web search engines, etc. [10].

By examining the elements of the eigenvector associated with the first mode, it is found that the tasks which require the greatest amount of work throughout the iteration process until a feasible solution is reached are associated with the largest values of the eigenvector (Tasks C-E, G, H, J, and L). It was also observed above that Task D, E, and $\mathrm{H}$ showed more work to be done on these tasks after one iteration, and confirmation of this result is in the fact that these tasks are most significant in the first mode (largest associated eigenvector values). This is indicative of the tighter coupling between these three tasks compared to others of lower dependency relationships, as well as being among the difficult tasks to complete (where additional work may be required initially). Summing the work vectors of the design process also indicates that Task $\mathrm{D}, \mathrm{E}$, and $\mathrm{H}$ require the most work to be done compared to the other tasks.

Table 2. Eigenvectors for Mode 1 and 2

\begin{tabular}{|c|c|c|c|}
\hline $\begin{array}{c}\text { Design } \\
\text { Task }\end{array}$ & Mode 1 & Mode 2 & Total Work \\
\hline C & 0.3248 & -0.0016 & 6.8804 \\
D & 0.3584 & -0.0150 & 7.5797 \\
E & 0.4137 & 0.2745 & 8.5905 \\
F & 0.1148 & -0.0914 & 3.0704 \\
G & 0.2777 & -0.6784 & 6.1058 \\
H & 0.4236 & -0.0784 & 8.7406 \\
I & 0.2934 & 0.6692 & 6.3960 \\
J & 0.2815 & -0.0008 & 6.1618 \\
K & 0.2633 & 0.0268 & 5.7197 \\
L & 0.3008 & -0.0344 & 6.5021 \\
\hline
\end{tabular}

An examination of the second mode shows that by far, the dominating task is Task I (evaluating Factors of Safety). Although the eigenvector value for Task I is somewhat significant in the first mode, it is much more significant in the second mode (by approximately twice as much); as such, the task can be considered part of a separate design mode, as opposed to being considered part of the first mode. In the design of the tire and wheel manipulating mechanism, students also consider factors of safety in their design, being required to stay within a prescribed range, while meeting other requirements. From the information given by the first and second mode, it can be said that there are two subprocesses - design and validation (Mode 1) and safety considerations (Mode 2).

The information provided by the eigenstructure analysis above would be primarily useful to instructors as a means of quantifying the relative difficulty of design term projects as well as to students in planning out design tasks for the project. For example, evaluating the design using dynamic simulation requires much work, but students can reduce the work involved at this stage (eg., require fewer simulations) by developing the improved concept early in the design process. At this preliminary stage, faster iterations may be necessary. Further, it should be noted that students in this project are restricted to using the available components of their Lego Mindstorms ${ }^{\circledR}$ kits (i.e., they cannot introduce new components). By better understanding this restriction, students can be more focused in developing the detailed design in CAD, determining better how 
components should be placed to efficiently come up with a design that could feasibly be built using the Lego Mindstorms ${ }^{\circledR}$ kit. This way, fewer iterations would be required to build and evaluate the prototype.

\section{Conclusions}

In this paper, existing product design and development methods were applied in evaluating the design process of a third-year engineering design project. Namely, an extension of the design structure matrix, the work transformation matrix, was used to show dependency strength between iterative tasks. From this matrix, the eigenstructure was determined and the most significant modes were examined to determine which tasks contribute the most work in the design process. Also, findings were confirmed by adding up the total work performed for each task to determine which tasks required the most work overall. The analysis is considered as being quite useful for quantifying the level of relative difficulty of assigned design term projects by engineering educators. It was also found to be useful to guide students in better planning their design process and where fewer and/or faster iterations are needed to efficiently come up with a feasible design. This analysis can be used for both existing, as well as introducing completely new design projects to students as well as to compare the level of challenge among different design projects.

\section{Acknowledgements}

The authors would like to thank the Natural Sciences and Engineering Research Council (NSERC) of Canada, General Motors Canada Limited (GMCL), and UOIT for financially supporting UOIT's Design Chair budget, part of which supported this research work. The authors are also grateful for the hardware and software provided by PACE (Partners for the Advancement of Collaborative Engineering Education).

\section{References}

[1] S.D. Eppinger, D.E. Whitney, R.P. Smith, and D.A. Gebala, "A Model-Based Method for Organizing Tasks in Product Development," Research in Engineering Design, Vol. 6, No. 1, 1994, pp. 1-13.

[2] R.P. Smith and S.D. Eppinger, "Identifying Controlling Features of Engineering Design Iteration," Management Science, Vol. 43, No. 3, March 1997, pp. 276-293.
[3] R.P. Smith and S.D. Eppinger, "A Predictive Model of Sequential Iteration in Engineering Design," Management Science, Vol. 43, No. 8, August 1997, pp. 1104-1120.

[4] P. Cronemyr, A.Ö. Rönnbäck, S.D.Eppinger, “A Decision Support Tool for Predicting the Impact of Development Process Improvements," Journal of Engineering Design, Vol. 12, No. 3, September 2001, pp. 177-199.

[5] D. Kim, "On Representations and Dynamic Analysis of Concurrent Engineering Design," Journal of Engineering Design, Vol. 18, No. 3, June 2001, pp. 265-277.

[6] K.L. Ong, S.G. Yee, and L.P. Khoo, "Homogeneous State-Space Representation of Concurrent Design," Journal of Engineering Design, Vol. 14, No. 2, June 2003, pp. 221-245.

[7] K.T. Ulrich and S.D. Eppinger, Product Design and Development, Fourth Edition, McGraw-Hill, 2008, pp. 333-358.

[8] D.V. Steward, "The Design Structure System: A Method for Managing the Design of Complex Systems" IEEE Transactions on Engineering Management, Vol. 28, No. 3, 1981, pp. 71-74.

[9] K. Ogata, Discrete-Time Control Systems, second edition, Prentice Hall Inc., 1995. pp. 74-288.

[10] U. Pillai, T. Suel, and S. Cha, "The PerronFrobenius Theorem - Some of its Applications," IEEE Signal Processing Magazine, Vol. 22, No. 2, March 2005, pp. 62-75. 